\title{
Indirect Dark Matter Searches with the ANTARES and KM3NeT Neutrino Telescopes
}

\section{Lopez-Coto ${ }^{1, *}$, S. Navas ${ }^{1}$ and J.D. Zornoza ${ }^{2}$ on behalf of the ANTARES ${ }^{\dagger}$ and $\mathrm{KM} 3 \mathrm{NeT}^{\ddagger}$ Collaborations}

(a complete list of authors can be found at the end of the proceedings)

${ }^{1}$ University of Granada

Dpto. de Física Teórica y del Cosmos \& C.A.F.P.E., Av. Hospicio 18071, Granada, Spain.

${ }^{2}$ IFIC - Instituto de Física Corpuscular

CSIC - Universitat de València,

c/ Catedrático José Beltrán, 2 E-46980 Paterna, Valencia, Spain.

E-mail: daniellc@ugr.es

Neutrino telescopes perform an indirect search for dark matter (DM) through its annihilation into standard model channels yielding neutrinos, for a broad range of WIMP masses. The ANTARES detector, anchored to the Mediterranean seabed at a depth of about $2500 \mathrm{~m}$, looks for a DM signal from two promising neutrino sources from WIMP annihilation: the Galactic Center and the Sun. We present the latest results on ANTARES indirect detection in a wide range of WIMP masses and decay channels, and give a future prospect on sensitivities of DM searches with the KM3NeT detector, the next-generation neutrino telescope, currently under deployment in the Mediterranean Sea. These experiments have specific advantages, complementary to other detection strategies, and can provide a smoking-gun signal. The geographical location of ANTARES and KM3NeT is particularly well suited for searches in the Galactic Center, allowing for the world-best sensitivity for WIMP annihilation.

\footnotetext{
*** The European Physical Society Conference on High Energy Physics (EPS-HEP2021), ***

*** 26-30 July 2021 ***

*** Online conference, jointly organized by Universität Hamburg and the research center DESY ***
}

\footnotetext{
${ }^{*}$ Speaker
} 


\section{Introduction}

Dark matter (DM) accounts for $26.4 \%$ of the critical density of the universe, but its nature remains unknown [1]. Among the diverse existing DM models, WIMPs (Weakly Interacting Massive Particles) are particularly interesting candidates. The hypothesis that WIMPs are unstable particles opens up the possibility to do indirect searches for WIMPs through the detection of Standard Model (SM) particles, like neutrinos, resulting from WIMP annihilation or decay. The cold and massive nature of WIMPs favors their accretion into celestial bodies, from which their signatures can be searched for. An alternative hypothesis for DM particles is called Secluded Dark Matter. This scenario is based on the idea that DM lives in the dark sector, and their annihilation to SM particles is only possible through a metastable mediator $(\phi)$, which afterwards decays to SM signatures [2-4]. The excellent pointing accuracy of the ANTARES and KM3NeT detectors (Fig. 1) makes them very well suited to perform these searches. Limits on the spin dependent (SD) and spin independent (SI) cross sections, for the solar searches, as well as on the thermally averaged cross sections [5], for the Galactic Center searches, are established.

This document is organized as follows. In section 2, the ANTARES and KM3NeT neutrino telescopes are briefly described. The ANTARES and KM3NeT WIMP searches towards the Sun and the Galactic Center are presented in section 3.1 and section 3.2, respectively. Section 4 ilustrates the results of the secluded DM scenario search.
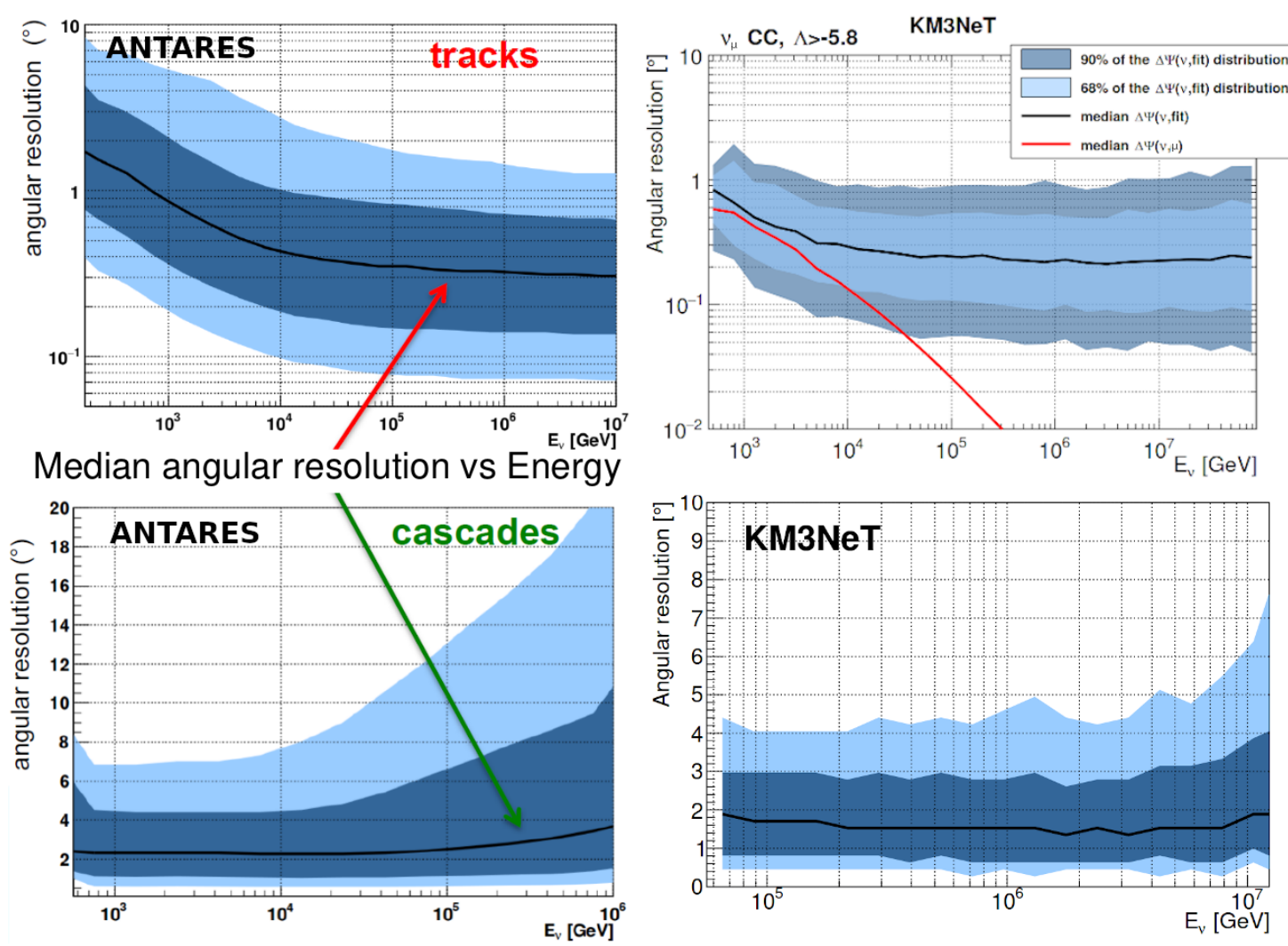

Figure 1: Median angular resolution in ANTARES (left) and KM3NeT (right) for reconstructed track (top) and shower (bottom) events. 


\section{The ANTARES and KM3NeT Neutrino Telescopes}

The ANTARES detector, anchored on the Mediterranean seabed $40 \mathrm{~km}$ offshore of Toulon, France, is the first undersea neutrino telescope [6]. The ANTARES full configuration, completed in 2008, comprises 12 detection lines separated 60-75 m and distributed in an octogonal layout. ANTARES has 295 storeys in total, distributed within the 12 detection lines and with a vertical spacing of about $14 \mathrm{~m}$. The $12^{\text {th }}$ line has 20 storeys and is completed with acoustic detection devices. Each storey contains a triad of optical modules (OMs) [7], housing a photomultiplier tube (PMT). The PMTs are pointing $45^{\circ}$ downward in order to optimize the detection of upgoing light from charged particles.

The KM3NeT detector (under deployment) [8] is the next generation undersea neutrino telescope. This infrastructure will consist of two main topologies of 115 strings $^{1}$, each string holds 18 digital optical modules (DOMs) and each DOM houses 31 3-inch PMTs. One of the configurations is referred to as ARCA (Astroparticle Research with Cosmics in the Abyss). The other one, more densely configured, is referred to as ORCA (Oscillation Research with Cosmics in the Abyss). ORCA is being installed offshore of Toulon, close to the actual site of ANTARES, while ARCA is being installed at Capo Passero, Italy.

Photons impinging in the sensitive area of the PMTs can induce a signal, called hit [9]. Each hit has associated a position, time and collected charge which are used to reconstruct the direction and energy of each event. The influence of the environmental noise (mainly by ${ }^{40} \mathrm{~K}$ and bioluminiscence) is reduced by different trigger algorithms [6].

The hard environmental conditions to which an undersea neutrino telescope is exposed may affect the trigger and data acquisition. Therefore, to correctly reproduce the response of the detector under these conditions, a Monte Carlo (MC) run-by-run strategy [10] is followed.
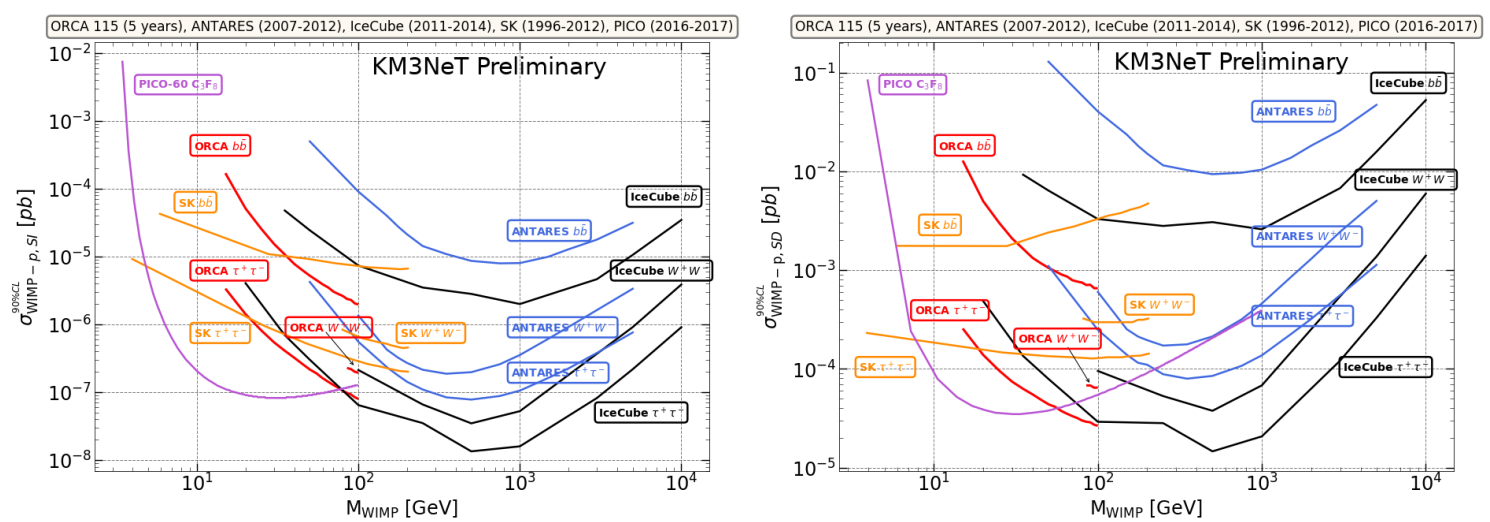

Figure 2: Sensitivities for Spin Independent (left) and Spin Dependent (right) WIMP-proton cross section derived from WIMP annihilation in the Sun, as function of the WIMP mass. The ANTARES limits (blue lines) [11] and the KM3NeT sensitivites (red lines) [12] are shown alongside the results of 3 years of IceCube data (black lines) [13], 1 year of PICO-60 $\mathrm{C}_{3} \mathrm{~F}_{8}$ data (purple line) [14] and 16 years of Super Kamiokande data (orange lines) [15], for comparison.

\footnotetext{
${ }^{1}$ The word string and detection lines are interchangeable throughout the text.
} 


\section{WIMP Dark Matter}

\subsection{Solar WIMP Dark Matter}

The Sun is a privileged place for searching for DM signatures given its proximity to the Earth. The dominant background on searches for DM in the Sun comes from atmospheric muons, atmospheric neutrinos and solar atmospheric neutrinos [20]. The ANTARES sensitivity to this last source of contamination has been studied for 11 years of ANTARES data and presented at the ICRC conference [21].

In 2016 ANTARES published an indirect search for solar WIMP DM particles with 5 years of data (2007-2012) [11], through three main annihilation channels (WIMP-WIMP $\rightarrow b \bar{b}, W^{+} W^{-}$ and $\tau^{+} \tau^{-}$). A new analysis with the full ANTARES data set is in progress. In 2019, KM3NeT performed a preliminary analysis on the potential of the full ORCA detector (115 detection lines) searching for neutrinos yielded by solar WIMP DM annihilations for 5 years of data taking [12]. In Fig. 2 these results from ANTARES and KM3NeT are shown. Equilibrium between annihilation and capture rates is assumed. ORCA improves the sensitivity to solar WIMP DM with respect to ANTARES by almost two orders of magnitude, being highly competitive with other experiments like IceCube within a similar data taking period.

\subsection{WIMP Dark Matter in the Galactic Center}

ANTARES and KM3NeT, being located in the northern hemisphere, have a privileged position to look to the galactic center (GC). Dark matter accumulated in the GC is distributed in halos, with a higher density of DM at the center. The spatial profile of the halo is described by the so called $J$-factor, which is function of the dark matter density $\rho$ integrated along the line of sight. In the analysis reported here, three different models for the $J$-factor have been tested: NFW [22], McMillan [23] and Burkert [24].

In 2020 the ANTARES search for DM in the GC using 11 years of data was published [25]. In the same year, a combined analysis using 9 years of ANTARES data and 3 years of IceCube data
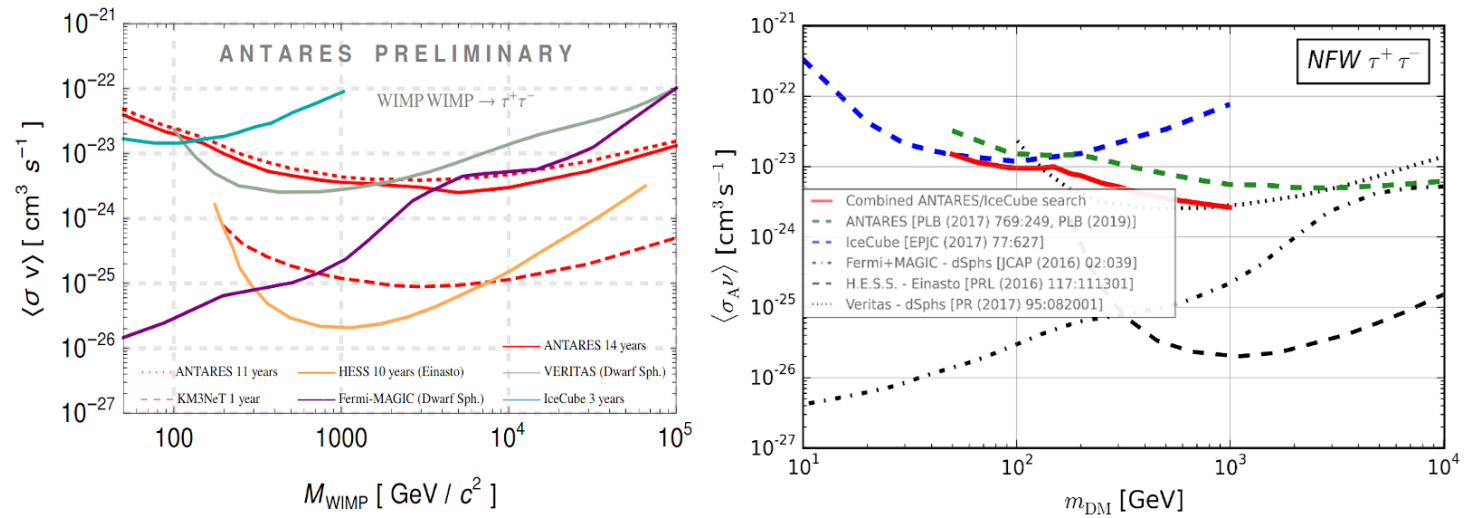

Figure 3: Upper limits at $90 \%$ C.L. on the thermally averaged cross-section for WIMP pair annihilation in the GC as a function of the WIMP mass for 14 years of ANTARES data, in comparison with IceCube [16], HESS [17], Veritas [18], Fermi-LAT+MAGIC [19] (left), and for the ANTARES-IceCube combined analysis (right). 
was also published [26]. In 2021, ANTARES and KM3NeT performed an analysis of DM in the GC [27], and compared the thermally averaged cross-section for WIMP annihilations between 14 years of ANTARES data (2007-2020) and the potential sensitivity of 1 year of full ARCA (221 lines), with the results of the same measurement from IceCube, HESS, Veritas and Fermi-LAT+MAGIC. The comparison between the 14 years of ANTARES data and the potential of 1 year of ARCA is presented on the left panel of Fig. 3. The great improvement on the sensitivity on the thermally averaged cross-section for WIMP pair annihilation of ARCA with respect to ANTARES is shown. Moreover, ARCA shows to be potentially competitive for DM searches in the GC. On the right side of Fig. 3, the ANTARES-IceCube combined search is plotted. This combined analysis shows an improvement with respect to searches carried out by each experiment independently.

\section{Secluded Dark Matter}

\subsection{Secluded Dark Matter in the Sun}

In secluded models, DM particles decay into SM particles through an unstable mediator. The first result on the search for secluded DM from the Sun with the ANTARES detector using 6 years of data (2007 - 2012) was published in 2016 [28] and is shown in Fig. 4. In this analysis, three different scenarios for a typical mediator mass of $1 \mathrm{GeV}$ were tested: (a) direct detection of dimuons produced by the decay of a long-lived mediator, (b) neutrino detection from the decaying dimuons produced by mediators that decayed before reaching the Earth, and (c) the detection of neutrinos decaying directly from mediators, for a mediator lifetime long enough to escape the solar medium.

The lifetime for the dimuon case has to be long enough to ensure that the mediator reaches the vicinity of the Earth. In contrast, for both neutrino cases, the lifetime of the mediator has to be long enough to escape the solar medium, but not so long that it decays before reaching the Earth's vicinity.
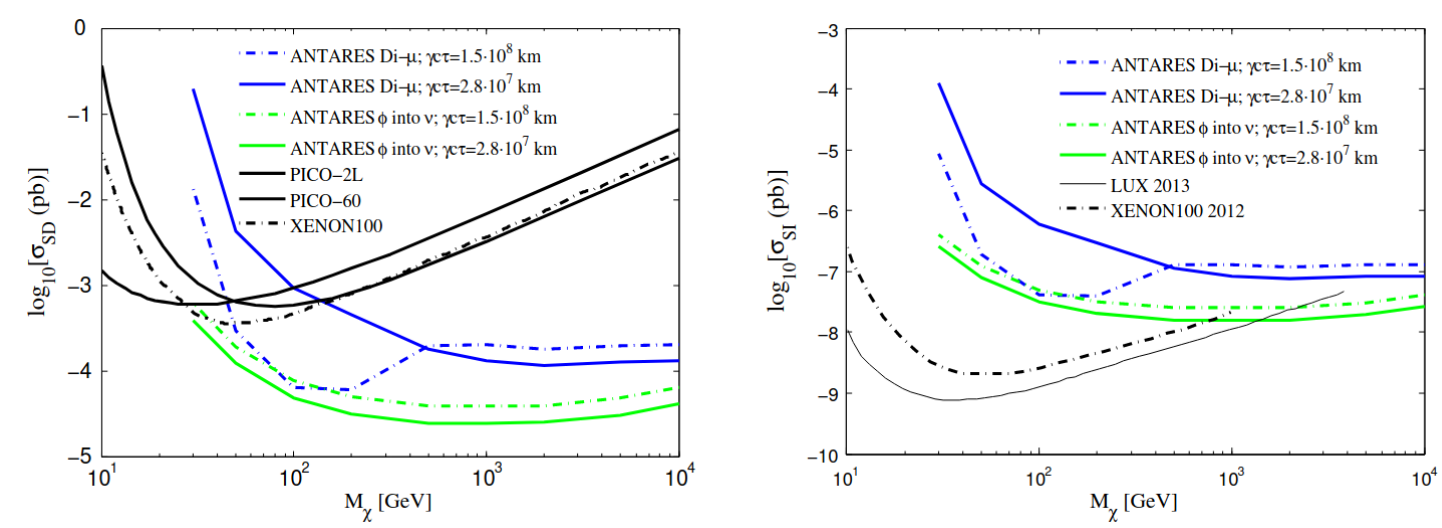

Figure 4: ANTARES upper limits at 90\% C.L. on WIMP-nucleon cross section, for the solar secluded DM scenario, as function of DM mass for SD (left) and SI (right) WIMP interactions. Two favourable mediator lifetimes are considered. $\gamma c \tau$ stands for the decay length of the mediator. The bounds from PICO [29-31], LUX [32] and XENON [33, 34] are also shown. 


\subsection{Secluded Dark Matter from the Galactic Center}

The Galactic Center is also suitable for secluded DM searches. Mediator particles decay in the Galactic Center producing SM particles that yield neutrinos via decays or showering. In 2020 ANTARES performed a search in the GC using 9 years of data (2007 - 2015) [35]. Mediator masses considered for the analysis were $50 \mathrm{GeV}, 250 \mathrm{GeV}$ and $1 \mathrm{TeV}$, and four final states SM particles: $\mu^{+} \mu^{-}, \tau^{+} \tau^{-}, b \bar{b}$ and $v \bar{v}$. No signal evidence was found. Limits on the thermally averaged annihilation cross section were established (Fig. 5).
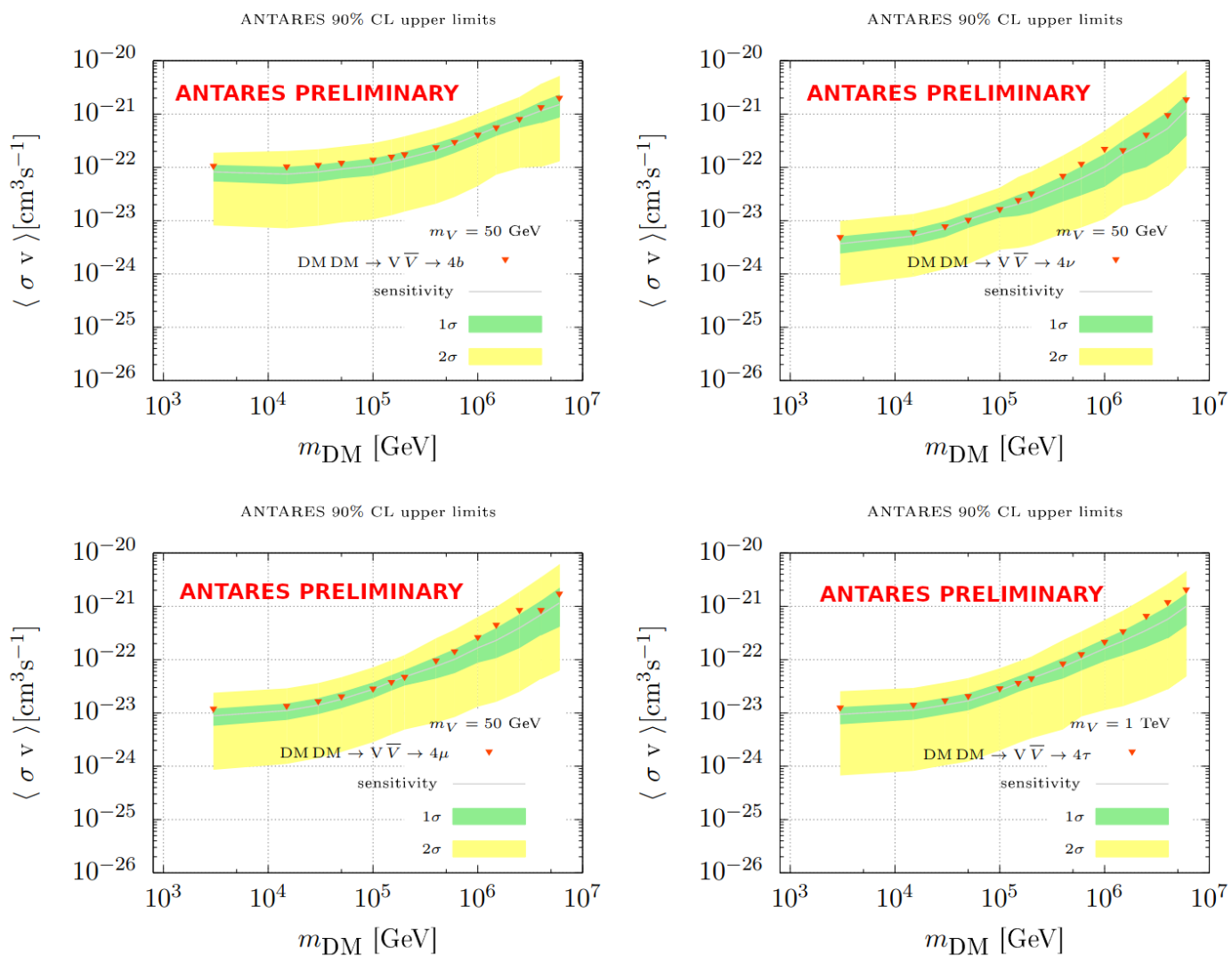

Figure 5: Upper limits at $90 \%$ C.L. on the thermally averaged annihilation cross section $\langle\sigma v\rangle$ for a mediator mass $\mathrm{m}_{\mathrm{V}}=50 \mathrm{GeV}$, with $1 \sigma$ and $2 \sigma$ containment bands, for four different final states [35] for secluded DM in the Galactic Center searches.

\section{Summary}

ANTARES and KM3NeT neutrino telescopes have been performing indirect dark matter searches for two different scenarios: WIMP, and Secluded dark matter particles. The Sun and the Galactic Center as sources for dark matter particles accretion have been explored. An unbinned (binned for the combined analysis) likelihood method seeking for dark matter signatures has been employed. No dark matter signal evidence has been found. Upper limits on the thermally averaged annihilation cross section, in the case of the GC, and on the WIMP-proton scattering cross section, for solar searches, have been established. KM3NeT has shown to be potentially competitive in both solar and galactic center DM searches scenarios, overcoming the actual ANTARES sensitivities. 


\section{References}

[1] P.A. Zyla et al., Review of particle physics, Prog. Theor. Exp. Phys. 2020 (2020).

[2] M. Pospelov et al., Secluded WIMP dark matter, Phys. Lett. B 662 (2008).

[3] N. Arkani-Hamed et al., A theory of dark matter, Phys. Rev. D 79 (2009).

[4] M. Pospelov et al., Astrophysical signatures of secluded dark matter, Phys. Lett. B 671 (2009).

[5] G. Bertone, ed., Particle Dark Matter, Cambridge University Press 10.1017/cbo9780511770739.

[6] M. Ageron et al., ANTARES: The First Undersea Neutrino Telescope, Nucl. Instrum. Meth. A 656 (2011).

[7] P. Amram et al., The ANTARES optical module, Nucl. Instrum. Meth. A 484 (2002).

[8] S. Adrián-Martínez et al., Letter of intent for KM3NeT 2.0, J. Phys. G: Nucl. Part. Phys. 43 (2016).

[9] J. Aguilar et al., Performance of the front-end electronics of the ANTARES neutrino telescope, Nucl. Instrum. Meth. A 622 (2010).

[10] A. Albert et al., Monte Carlo simulations for the ANTARES underwater neutrino telescope, J. Cosmol. Astropart. Phys. 2021 (2021).

[11] S. Adrián-Martínez et al., Limits on dark matter annihilation in the sun using the ANTARES neutrino telescope, Phys. Lett. B 759 (2016).

[12] S. Navas et al., Dark matter searches from the Sun with the KM3NeT-ORCA detector, in Proceedings of 36th International Cosmic Ray Conference — PoS(ICRC2019), Sissa Medialab, 2019.

[13] M.G. Aartsen et al., Search for annihilating dark matter in the sun with 3 years of IceCube data: IceCube collaboration, Eur. Phys. J. C 77 (2017).

[14] C. Amole et al., Dark matter search results from the PICO-60 $C_{3} F_{8}$ bubble chamber, Phys. Rev. Lett. 118 (2017).

[15] K. Frankiewicz, Searching for dark matter annihilation into neutrinos with Super-Kamiokande, in The Meeting of the American Physical Society Division of Particles and Fields (DPF 2015): Ann Arbor, Michigan, August 4-8, 2015, https://arxiv.org/abs/1510.07999.

[16] M.G. Aartsen et al., Search for neutrinos from dark matter self-annihilations in the center of the milky way with 3 years of IceCube/DeepCore, Eur. Phys. J. C 77 (2017). 
[17] H. Abdallah et al., Search for dark matter annihilations towards the inner galactic halo from 10 years of observations with H.E.S.S., Phys. Rev. Lett. 117 (2016).

[18] S. Archambault et al., Dark matter constraints from a joint analysis of dwarf spheroidal galaxy observations with VERITAS, Phys. Rev. D 95 (2017).

[19] M.L. Ahnen et al., Limits to dark matter annihilation cross-section from a combined analysis of MAGIC and fermi-LAT observations of dwarf satellite galaxies, J. Cosmol. Astropart. Phys. 2016 (2016).

[20] J. Edsjö et al., Neutrinos from cosmic ray interactions in the Sun, J. Cosmol. Astropart. Phys. 2017 (2017).

[21] D. Lopez-Coto et al., Solar atmospheric neutrinos searches with ANTARES neutrino telescope, in Proceedings of 37th International Cosmic Ray Conference - PoS(ICRC2021), vol. 395, Sissa Medialab, 2021.

[22] J.F. Navarro et al., The structure of cold dark matter halos, Astrophys. J. 462 (1996).

[23] P.J. McMillan, The mass distribution and gravitational potential of the milky way, Mon. Not. R. Astron. Soc. 465 (2016).

[24] A. Burkert, The structure of dark matter halos in dwarf galaxies, Astrophys. J. 447 (1995).

[25] A. Albert et al., Search for dark matter towards the galactic centre with 11 years of ANTARES data, Phys. Lett. B $\mathbf{8 0 5}$ (2020).

[26] A. Albert et al., Combined search for neutrinos from dark matter self-annihilation in the galactic centre with ANTARES and IceCube, Phys. Rev. D 102 (2020).

[27] S.R. Gozzini et al., Indirect dark matter searches with neutrinos from the galactic centre region with the ANTARES and KM3NeT telescopes, in Proceedings of 37th International Cosmic Ray Conference — PoS(ICRC2021), Sissa Medialab, 2021.

[28] S. Adrián-Martínez et al., A search for secluded dark matter in the sun with the ANTARES neutrino telescope, J. Cosmol. Astropart. Phys. 2016 (2016).

[29] C. Amole et al., Dark matter search results from the PICO-2L $C_{3} F_{8}$ bubble chamber, Phys. Rev. Lett. 114 (2015).

[30] C. Amole et al., Improved dark matter search results from PICO-2L Run 2, Phys. Rev. D 93 (2016).

[31] C. Amole et al., Dark matter search results from the PICO-60 CF $\mathrm{CF}_{3}$ bubble chamber, Phys. Rev. D 93 (2016).

[32] D.S. Akerib et al., First results from the LUX dark matter experiment at the Sanford Underground Research Facility, Phys. Rev. Lett. 112 (2014). 
[33] E. Aprile et al., Dark matter results from 225 live days of XENON100 data, Phys. Rev. Lett. 109 (2012).

[34] E. Aprile et al., Limits on spin-dependent wimp-nucleon cross sections from 225 live days of XENON100 data, Phys. Rev. Lett. 111 (2013).

[35] F. Sala et al., Search for heavy secluded dark matter with ANTARES, in XXIX International Conference On Neutrino Physics and Astrophysics. Neutrino 2020, Zenodo, 2020.

\section{Full Authors List: ANTARES Collaboration $\dagger$}

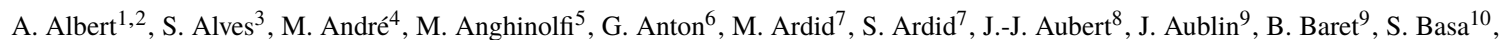
B. Belhorma ${ }^{11}$, M. Bendahman ${ }^{9}, 12$, V. Bertin ${ }^{8}$, S. Biagi ${ }^{13}$, M. Bissinger ${ }^{6}$, J. Boumaaza ${ }^{12}$, M. Bouta ${ }^{14}$, M.C. Bouwhuis ${ }^{15}$, H. Brânzaş ${ }^{16}$, R. Bruijn ${ }^{15,17}$, J. Brunner ${ }^{8}$, J. Busto ${ }^{8}$, B. Caiffi ${ }^{5}$, A. Capone ${ }^{18,19}$, L. Caramete ${ }^{16}$, J. Carr ${ }^{8}$, V. Carretero ${ }^{3}$, S. Celli ${ }^{18,19}$, M. Chabab ${ }^{20}$, T. N. Chau ${ }^{9}$, R. Cherkaoui El Moursli ${ }^{12}$, T. Chiarusi ${ }^{21}$, M. Circella ${ }^{22}$, A. Coleiro ${ }^{9}$, M. Colomer-Molla ${ }^{9,3}$, R. Coniglione ${ }^{13}$, P. Coyle ${ }^{8}$, A. Creusot $^{9}$, A. F. Díaz ${ }^{23}$, G. de Wasseige ${ }^{9}$, A. Deschamps ${ }^{24}$, C. Distefano ${ }^{13}$, I. Di Palma ${ }^{18,19}$, A. Domi ${ }^{15,17}$, C. Donzaud ${ }^{9,25}$, D. Dornic ${ }^{8}$, D. Drouhin ${ }^{1,2}$, T. Eberl ${ }^{6}$, T. van Eeden ${ }^{15}$, D. van Eijk ${ }^{15}$, N. El Khayati ${ }^{12}$, A. Enzenhöfer ${ }^{8}$, P. Fermani ${ }^{18,19}$, G. Ferrara ${ }^{13}$, F. Filippini ${ }^{21,26}$, L.A. Fusco ${ }^{8}$, Y. Gatelet ${ }^{9}$, P. Gay ${ }^{27,9}$, H. Glotin ${ }^{28}$, R. Gozzini ${ }^{3}$, R. Gracia Ruiz ${ }^{15}$, K. Graf ${ }^{6}$, C. Guidi ${ }^{5}, 29$, S. Hallmann ${ }^{6}$, H. van Haren ${ }^{30}$, A.J. Heijboer ${ }^{15}$, Y. Hello ${ }^{24}$, J.J. Hernández-Rey ${ }^{3}$, J. Hößl ${ }^{6}$, J. Hofestädt ${ }^{6}$, F. Huang ${ }^{8}$, G. Illuminati ${ }^{9}, 21,26$, C.W James ${ }^{31}$, B. Jisse-Jung ${ }^{15}$, M. de Jong ${ }^{15,32}$, P. de Jong ${ }^{15}$, M. Kadler ${ }^{33}$, O. Kalekin ${ }^{6}$, U. Katz ${ }^{6}$, N.R. Khan-Chowdhury ${ }^{3}$, A. Kouchner ${ }^{9}$, I. Kreykenbohm ${ }^{34}$, V. Kulikovskiy ${ }^{5,36}$, R. Lahmann ${ }^{6}$, R. Le Breton ${ }^{9}$, D. Lefèvre ${ }^{35}$, E. Leonora ${ }^{36}$, G. Levi ${ }^{21,26}$, M. Lincetto ${ }^{8}$, D. Lopez-Coto ${ }^{37}$, S. Loucatos ${ }^{38,9}$, L. Maderer ${ }^{9}$, J. Manczak ${ }^{3}$, M. Marcelin ${ }^{10}$, A. Margiotta ${ }^{21,26}$, A. Marinelli ${ }^{39}$, J.A. Martínez-Mora ${ }^{7}$, K. Melis ${ }^{15,17}$, P. Migliozzi ${ }^{39}$, A. Moussa ${ }^{14}$, R. Muller ${ }^{15}$, L. Nauta ${ }^{15}$, S. Navas ${ }^{37}$, E. Nezri ${ }^{10}$, B. O' Fearraigh ${ }^{15}$, A. Păun ${ }^{16}$, G.E. Păvălaş ${ }^{16}$, C. Pellegrino ${ }^{21,40,41}$, M. Perrin-Terrin ${ }^{8}$, V. Pestel ${ }^{15}$, P. Piattelli ${ }^{13}$, C. Pieterse ${ }^{3}$, C. Poirè ${ }^{7}$, V. Popa ${ }^{16}$, T. Pradier ${ }^{1}$, N. Randazzo ${ }^{36}$, S. Reck $^{6}$, G. Riccobene ${ }^{13}$, A. Romanov ${ }^{5,29}$, A. Sánchez-Losa ${ }^{3,22}$, F. Salesa Greus ${ }^{3}$, D. F. E. Samtleben ${ }^{15,32}$, M. Sanguineti ${ }^{5,29}$, P. Sapienza ${ }^{13}$, J. Schnabel ${ }^{6}$, J. Schumann ${ }^{6}$, F. Schüssler ${ }^{38}$, M. Spurio ${ }^{21,26}$, Th. Stolarczyk ${ }^{38}$, M. Taiuti ${ }^{5,29}$, Y. Tayalati ${ }^{12}$, S.J. Tingay ${ }^{31}$, B. Vallage ${ }^{38,9}$, V. Van Elewyck ${ }^{9,41}$, F. Versari ${ }^{21,26,9}$, S. Viola ${ }^{13}$, D. Vivolo ${ }^{39,43}$, J. Wilms ${ }^{34}$, S. Zavatarelli ${ }^{5}$, A. Zegarelli ${ }^{18,19}$, J.D. Zornoza ${ }^{3}$, and J. Zúñiga ${ }^{3}$

${ }^{1}$ Université de Strasbourg, CNRS, IPHC UMR 7178, F-67000 Strasbourg, France. ${ }^{2}$ Université de Haute Alsace, F-68100 Mulhouse, France. ${ }^{3}$ IFIC - Instituto de Física Corpuscular (CSIC - Universitat de València) c/ Catedrático José Beltrán, 2 E-46980 Paterna, Valencia, Spain. ${ }^{4}$ Technical University of Catalonia, Laboratory of Applied Bioacoustics, Rambla Exposició, 08800 Vilanova i la Geltrú, Barcelona, Spain. ${ }^{5}$ INFN - Sezione di Genova, Via Dodecaneso 33, 16146 Genova, Italy. ${ }^{6}$ Friedrich-Alexander-Universität Erlangen-Nürnberg, Erlangen Centre for Astroparticle Physics, Erwin-Rommel-Str. 1, 91058 Erlangen, Germany. ${ }^{7}$ Institut d'Investigació per a la Gestió Integrada de les Zones Costaneres (IGIC) - Universitat Politècnica de València. C/ Paranimf 1, 46730 Gandia, Spain. ${ }^{8}$ Aix Marseille Univ, CNRS/IN2P3, CPPM, Marseille, France. ${ }^{9}$ Université de Paris, CNRS, Astroparticule et Cosmologie, F-75013 Paris, France. ${ }^{10}$ Aix Marseille Univ, CNRS, CNES, LAM, Marseille, France. ${ }^{11}$ National Center for Energy Sciences and Nuclear Techniques, B.P.1382, R. P.10001 Rabat, Morocco. ${ }^{12}$ University Mohammed V in Rabat, Faculty of Sciences, 4 av. Ibn Battouta, B.P. 1014, R.P. 10000 Rabat, Morocco. ${ }^{13}$ INFN - Laboratori Nazionali del Sud (LNS), Via S. Sofia 62, 95123 Catania, Italy. ${ }^{14}$ University Mohammed I, Laboratory of Physics of Matter and Radiations, B.P.717, Oujda 6000, Morocco. ${ }^{15}$ Nikhef, Science Park, Amsterdam, The Netherlands. ${ }^{16}$ Institute of Space Science, RO-077125 Bucharest, Măgurele, Romania. ${ }^{17}$ Universiteit van Amsterdam, Instituut voor Hoge-Energie Fysica, Science Park 105, 1098 XG Amsterdam, The Netherlands. ${ }^{18}$ INFN - Sezione di Roma, P.le Aldo Moro 2, 00185 Roma, Italy. ${ }^{19}$ Dipartimento di Fisica dell’Università La Sapienza, P.le Aldo Moro 2, 00185 Roma, Italy. ${ }^{20}$ LPHEA, Faculty of Science - Semlali, Cadi Ayyad University, P.O.B. 2390, Marrakech, Morocco. ${ }^{21}$ INFN - Sezione di Bologna, Viale Berti-Pichat 6/2, 40127 Bologna, Italy. ${ }^{22}$ INFN - Sezione di Bari, Via E. Orabona 4, 70126 Bari, Italy. ${ }^{23}$ Department of Computer Architecture and Technology/CITIC, University of Granada, 18071 Granada, Spain. ${ }^{24}$ Géoazur, UCA, CNRS, IRD, Observatoire de la Côte d'Azur, Sophia Antipolis, France. ${ }^{25}$ Université Paris-Sud, 91405 Orsay Cedex, France. ${ }^{26}$ Dipartimento di Fisica e Astronomia dell'Università, Viale Berti Pichat 6/2, 40127 Bologna, Italy. ${ }^{27}$ Laboratoire de Physique Corpusculaire, Clermont Université, Université Blaise Pascal, CNRS/IN2P3, BP 10448, F-63000 Clermont-Ferrand, France. ${ }^{28}$ LIS, UMR Université de Toulon, Aix Marseille Université, CNRS, 83041 Toulon, France. ${ }^{29}$ Dipartimento di Fisica dell'Università, Via Dodecaneso 33, 16146 Genova, Italy. ${ }^{30}$ Royal Netherlands Institute for Sea Research (NIOZ), Landsdiep 4, 1797 SZ 't Horntje (Texel), the Netherlands. ${ }^{31}$ International Centre for Radio Astronomy Research - Curtin University, Bentley, WA 6102, Australia. ${ }^{32}$ Huygens-Kamerlingh Onnes Laboratorium, Universiteit Leiden, The Netherlands. ${ }^{33}$ Institut für Theoretische Physik und Astrophysik, Universität Würzburg, Emil-Fischer Str. 31, 97074 Würzburg, Germany. ${ }^{34}$ Dr. Remeis-Sternwarte and ECAP, Friedrich-Alexander-Universität Erlangen-Nürnberg, Sternwartstr. 7, 96049 Bamberg, Germany. ${ }^{35}$ Mediterranean Institute of Oceanography (MIO), Aix-Marseille University, 13288, Marseille, Cedex 9, France; Université du Sud Toulon-Var, CNRS-INSU/IRD UM 110, 83957, La Garde Cedex, France. ${ }^{36}$ INFN - Sezione di Catania, Via S. Sofia 64, 95123 Catania, Italy. ${ }^{37}$ Dpto. de Física Teórica y del Cosmos \& C.A.F.P.E., University of Granada, 18071 Granada, Spain. ${ }^{38}$ IRFU, 
CEA, Université Paris-Saclay, F-91191 Gif-sur-Yvette, France. ${ }^{39}$ INFN - Sezione di Napoli, Via Cintia 80126 Napoli, Italy. ${ }^{40}$ Museo Storico della Fisica e Centro Studi e Ricerche Enrico Fermi, Piazza del Viminale 1, 00184, Roma. ${ }^{41}$ INFN - CNAF, Viale C. Berti Pichat 6/2, 40127, Bologna. ${ }^{42}$ Institut Universitaire de France, 75005 Paris, France. ${ }^{43}$ Dipartimento di Fisica dell'Università Federico II di Napoli, Via Cintia 80126, Napoli, Italy.

\section{Full Author List: KM3NeT Collaboration}

M. Ageron ${ }^{1}$, S. Aiello ${ }^{2}$, A. Albert ${ }^{3,55}$, M. Alshamsi ${ }^{4}$, S. Alves Garre ${ }^{5}$,Z. Aly ${ }^{1}$, A. Ambrosone ${ }^{6,7}$, F. Ameli ${ }^{8}$, M. Andre ${ }^{9}$, G. Androulakis ${ }^{10}$, M. Anghinolf ${ }^{11}$, M. Anguita ${ }^{12}$, G. Anton ${ }^{13}$, M. Ardid ${ }^{14}$, S. Ardid $^{14}$, W. Assal ${ }^{1}$, J. Aublin ${ }^{4}$, C. Bagatelas ${ }^{10}$, B. Baret ${ }^{4}$, S. Basegmez du Pree ${ }^{15}$, M. Bendahman ${ }^{4,16}$, F. Benfenati ${ }^{17,18}$, E. Berbee ${ }^{15}$, A. M. van den Berg ${ }^{19}$, V. Bertin ${ }^{1}$, S. Beurthey ${ }^{1}$, V. van Beveren ${ }^{15}$, S. Biagi ${ }^{20}$, M. Billault ${ }^{1}$, M. Bissinger ${ }^{13}$, M. Boettcher ${ }^{21}$, M. Bou Cabo ${ }^{22}$, J. Boumaaza ${ }^{16}$, M. Bouta ${ }^{23}$, C. Boutonnet ${ }^{4}$, G. Bouvet ${ }^{24}$, M. Bouwhuis ${ }^{15}$, C. Bozza ${ }^{25}$, H.Brânzaş ${ }^{26}$, R. Bruijn ${ }^{15,27}$, J. Brunner ${ }^{1}$, R. Bruno ${ }^{2}$, E. Buis ${ }^{28}$, R. Buompane ${ }^{6,29}$, J. Busto ${ }^{1}$, B. Caiffi ${ }^{11}$, L. Caillat ${ }^{1}$, D. Calvo ${ }^{5}$, S. Campion ${ }^{30,8}$, A. Capone ${ }^{30,8}$, H. Carduner ${ }^{24}$, V. Carretero ${ }^{5}$, P. Castaldi ${ }^{17,31}$, S. Celli ${ }^{30,8}$, R. Cereseto ${ }^{11}$, M. Chabab ${ }^{32}$, C. Champion ${ }^{4}$, N. Chau ${ }^{4}$, A. Chen ${ }^{33}$, S. Cherubini $^{20,34}$, V. Chiarella ${ }^{35}$, T. Chiarusi ${ }^{17}$, M. Circella ${ }^{36}$, R. Cocimano ${ }^{20}$, J. A. B. Coelho ${ }^{4}$, A. Coleiro ${ }^{4}$, M. Colomer Molla ${ }^{4,5}$, S. Colonges ${ }^{4}$, R. Coniglione ${ }^{20}$, A. Cosquer ${ }^{1}$, P. Coyle ${ }^{1}$, M. Cresta ${ }^{11}$, A. Creusot ${ }^{4}$, A. $\mathrm{Cruz}^{37}$, G. Cuttone ${ }^{20}$, A. D’Amico ${ }^{15}$, R. Dallier ${ }^{24}$, B. De Martino ${ }^{1}$, M. De Palma ${ }^{36,38}$, I. Di Palma ${ }^{30,8}$, A. F. Díaz ${ }^{12}$, D. DiegoTortosa $^{14}$, C. Distefano ${ }^{20}$, A. Domi ${ }^{15,27}$, C. Donzaud ${ }^{4}$, D. Dornic ${ }^{1}$, M. Dörr ${ }^{39}$, D. Drouhin ${ }^{3,55}$, T. Eberl ${ }^{13}$, A. Eddyamoui ${ }^{16}$, T. van Eeden ${ }^{15}$, D. van Eijk ${ }^{15}$, I. El Bojaddaini ${ }^{23}$, H. Eljarrari ${ }^{16}$, D. Elsaesser ${ }^{39}$, A. Enzenhöfer ${ }^{1}$, V. Espinosa ${ }^{14}$, P. Fermani ${ }^{30,8}$, G. Ferrara ${ }^{20,34}$, M. D. Filipovićc ${ }^{40}$, F. Filippini ${ }^{17,18}$, J. Fransen ${ }^{15}$, L. A. Fusco ${ }^{1}$, D. Gajanana ${ }^{15}$, T. Gal ${ }^{13}$, J. García Méndez ${ }^{14}$, A. Garcia Soto $^{5}$, E. Garçon ${ }^{1}$, F. Garufi ${ }^{6,7}$, C. Gatius ${ }^{15}$, N. Geißelbrecht ${ }^{13}$, L. Gialanella ${ }^{6,29}$, E. Giorgio ${ }^{20}$, S. R. Gozzini ${ }^{5}$, R. Gracia ${ }^{15}$, K. Graf ${ }^{13}$, G. Grella ${ }^{41}$, D. Guderian ${ }^{56}$, C. Guidi ${ }^{11,42}$, B. Guillon ${ }^{43}$, M. Gutiérrez ${ }^{44}$, J. Haefner ${ }^{13}$, S. Hallmann ${ }^{13}$, H. Hamdaoui ${ }^{16}$, H. van Haren ${ }^{45}$, A. Heijboer ${ }^{15}$, A. Hekalo ${ }^{39}$, L. Hennig ${ }^{13}$, S. Henry ${ }^{1}$, J. J. Hernández-Rey ${ }^{5}$, J. Hofestädt ${ }^{13}$, F. Huang ${ }^{1}$, W. Idrissi Ibnsalih ${ }^{6,29}$, A. Ilioni ${ }^{4}$, G. Illuminati ${ }^{17,18,4}$, C. W. James ${ }^{37}$, D. Janezashvili ${ }^{46}$, P. Jansweijer ${ }^{15}$, M. de Jong ${ }^{15,47}$, P. de Jong ${ }^{15,27}$, B. J. Jung ${ }^{15}$, M. Kadler ${ }^{39}$, P. Kalaczyński ${ }^{48}$, O. Kalekin ${ }^{13}$, U. F. Katz ${ }^{13}$, F. Kayzel ${ }^{15}$, P. Keller ${ }^{1}$, N. R. Khan Chowdhury ${ }^{5}$, G. Kistauri ${ }^{46}$, F. van der Knaap ${ }^{28}$, P. Kooijman ${ }^{27,57}$, A. Kouchner, ${ }^{49}$, M. Kreter ${ }^{21}$, V. Kulikovskiy ${ }^{11}$, M. Labalme ${ }^{43}$, P. Lagier ${ }^{1}$, R. Lahmann ${ }^{13}$, P. Lamare ${ }^{1}$, M. Lamoureux $^{24}$, G. Larosa ${ }^{20}$, C. Lastoria ${ }^{1}$, J. Laurence ${ }^{1}$, A. Lazo ${ }^{5}$, R. Le Breton ${ }^{4}$, E. Le Guirriec ${ }^{1}$, S. Le Stum ${ }^{1}$, G. Lehaut ${ }^{43}$, O. Leonardi ${ }^{20}$, F. Leone ${ }^{20,34}$, E. Leonora ${ }^{2}$, C. Lerouvillois ${ }^{1}$, J. Lesrel ${ }^{4}$, N. Lessing ${ }^{13}$, G. Levi ${ }^{17,18}$, M. Lincetto ${ }^{1}$, M. Lindsey Clark ${ }^{4}$, T. Lipreau ${ }^{24}$, C. LLorens Alvarez ${ }^{14}$, A. Lonardo ${ }^{8}$, F. Longhitano ${ }^{2}$, D. Lopez-Coto ${ }^{44}$, N. Lumb ${ }^{1}$, L. Maderer ${ }^{4}$, J. Majumdar ${ }^{15}$, J. Mańczak ${ }^{5}$, A. Margiotta ${ }^{17,18}$, A. Marinelli ${ }^{6}$, A. Marini ${ }^{1}$, C. Markou $^{10}$, L. Martin ${ }^{24}$, J. A. Martínez-Mora ${ }^{14}$, A. Martini ${ }^{35}$, F. Marzaioli ${ }^{6,29}$,

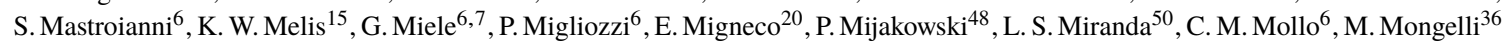
A. Moussa ${ }^{23}$, R. Muller ${ }^{15}$, P. Musico ${ }^{11}$, M. Musumeci ${ }^{20}$, L. Nauta ${ }^{15}$, S. Navas ${ }^{44}$, C. A. Nicolau ${ }^{8}$, B. Nkosi ${ }^{33}$, B. Ó Fearraigh ${ }^{15,27}$, M. O'Sullivan ${ }^{37}$, A. Orlando ${ }^{20}$, G. Ottonello ${ }^{11}$, S. Ottonello ${ }^{11}$, J. Palacios González ${ }^{5}$, G. Papalashvilili , R. Papaleo ${ }^{20}$, C. Pastore ${ }^{36}$, A. M. Păun ${ }^{26}$, G. E. Păvălaşş, G. Pellegrini ${ }^{17}$, C. Pellegrino ${ }^{18,58}$, M. Perrin-Terrin ${ }^{1}$, V. Pestel ${ }^{15}$, P. Piattelli ${ }^{20}$, C. Pieterse ${ }^{5}$, O. Pisanti ${ }^{6,7}$, C. Poirè ${ }^{14}$, V. Popa ${ }^{26}$, T. Pradier $^{3}$, F. Pratolongo ${ }^{11}$, I. Probst ${ }^{13}$, G. Pühlhofer ${ }^{51}$, S. Pulvirenti ${ }^{20}$, G. Quéméner ${ }^{43}$, N. Randazzo ${ }^{2}$, A. Rapicavoli ${ }^{34}$, S. Razzaque ${ }^{50}$, D. Real ${ }^{5}$, S. Reck ${ }^{13}$, G. Riccobene ${ }^{20}$, L. Rigalleau ${ }^{24}$, A. Romanov ${ }^{11,42}$, A. Rovelli ${ }^{20}$, J. Royon ${ }^{1}$, F. Salesa Greus ${ }^{5}$, D. F. E. Samtleben ${ }^{15,47}$, A. Sánchez Losa ${ }^{36,5}$, M. Sanguineti ${ }^{11,42}$, A. Santangelo ${ }^{51}$, D. Santonocito ${ }^{20}$, P. Sapienza ${ }^{20}$, J. Schmelling ${ }^{15}$, J. Schnabel ${ }^{13}$, M. F. Schneider ${ }^{13}$, J. Schumann ${ }^{13}$, H. M. Schutte ${ }^{21}$, J. Seneca ${ }^{15}$, I. Sgura ${ }^{36}$, R. Shanidze ${ }^{46}$, A. Sharma ${ }^{52}$, A. Sinopoulou ${ }^{10}$, B. Spisso ${ }^{41,6}$, M. Spurio ${ }^{17,18}$, D. Stavropoulos ${ }^{10}$, J. Steijger ${ }^{15}$, S. M. Stellacci ${ }^{41,6}$, M. Taiuti ${ }^{11,42}$, F. Tatone ${ }^{36}$, Y. Tayalati ${ }^{16}$, E. Tenllado ${ }^{44}$, D. Tézier ${ }^{1}$, T. Thakore ${ }^{5}$, S. Theraube ${ }^{1}$, H. Thiersen $^{21}$, P. Timmer $^{15}$, S. Tingay ${ }^{37}$, S. Tsagkli ${ }^{10}$, V. Tsourapis ${ }^{10}$, E. Tzamariudaki ${ }^{10}$, D. Tzanetatos ${ }^{10}$, C. Valieri ${ }^{17}$, V. Van Elewyck ${ }^{4,49}$, G. Vasileiadis ${ }^{53}$, F. Versari ${ }^{17,18}$, S. Viola ${ }^{20}$, D. Vivolo ${ }^{6,29}$, G. de Wasseige ${ }^{4}$, J. Wilms ${ }^{54}$, R. Wojaczyński ${ }^{48}$, E. de Wolf ${ }^{15,27}$, T. Yousfi ${ }^{23}$, S. Zavatarelli ${ }^{11}$, A. Zegarelli ${ }^{30,8}$, D. Zito ${ }^{20}$, J. D. Zornoza ${ }^{5}$, J. Zúñiga ${ }^{5}$, N. Zywucka ${ }^{21}$.

${ }^{1}$ Aix Marseille Univ, CNRS/IN2P3, CPPM, Marseille, France.

${ }^{2}$ INFN, Sezione di Catania, Via Santa Sofia 64, Catania, 95123 Italy.

${ }^{3}$ Université de Strasbourg, CNRS, IPHC UMR 7178, F-67000 Strasbourg, France.

${ }^{4}$ Université de Paris, CNRS, Astroparticule et Cosmologie, F-75013 Paris, France.

${ }^{5}$ IFIC - Instituto de Física Corpuscular (CSIC - Universitat de València), c/Catedrático José Beltrán, 2, 46980 Paterna, Valencia, Spain. ${ }^{6}$ INFN, Sezione di Napoli, Complesso Universitario di Monte S. Angelo, Via Cintia ed. G, Napoli, 80126 Italy.

${ }^{7}$ Università di Napoli "Federico II", Dip. Scienze Fisiche "E. Pancini”, Complesso Universitario di Monte S. Angelo, Via Cintia ed. G, Napoli, 80126 Italy.

${ }^{8}$ INFN, Sezione di Roma, Piazzale Aldo Moro 2, Roma, 00185 Italy.

${ }^{9}$ Universitat Politècnica de Catalunya, Laboratori d'Aplicacions Bioacústiques, Centre Tecnològic de Vilanova i la Geltrú, Avda. Rambla Exposició, s/n, Vilanova i la Geltrú, 08800 Spain.

${ }^{10}$ NCSR Demokritos, Institute of Nuclear and Particle Physics, Ag. Paraskevi Attikis, Athens, 15310 Greece.

${ }^{11}$ INFN, Sezione di Genova, Via Dodecaneso 33, Genova, 16146 Italy.

${ }^{12}$ University of Granada, Dept. of Computer Architecture and Technology/CITIC, 18071 Granada, Spain.

${ }^{13}$ Friedrich-Alexander-Universität Erlangen-Nürnberg, Erlangen Centre for Astroparticle Physics, Erwin-Rommel-Straße 1, 91058 Erlangen, Germany.

\footnotetext{
${ }^{2}$ also at Dipartimento di Fisica, INFN Sezione di Padova and Università di Padova, I-35131, Padova, Italy
} 
${ }^{14}$ Universitat Politècnica de València, Instituto de Investigación para la Gestión Integrada de las Zonas Costeras, C/ Paranimf, 1, Gandia, 46730 Spain.

${ }^{15}$ Nikhef, National Institute for Subatomic Physics, PO Box 41882, Amsterdam, 1009 DB Netherlands.

${ }^{16}$ University Mohammed V in Rabat, Faculty of Sciences, 4 av. Ibn Battouta, B.P. 1014, R.P. 10000 Rabat, Morocco.

${ }^{17}$ INFN, Sezione di Bologna, v.le C. Berti-Pichat, 6/2, Bologna, 40127 Italy.

${ }^{18}$ Università di Bologna, Dipartimento di Fisica e Astronomia, v.le C. Berti-Pichat, 6/2, Bologna, 40127 Italy.

${ }^{19}$ KVI-CART University of Groningen, Groningen, the Netherlands.

${ }^{20}$ INFN, Laboratori Nazionali del Sud, Via S. Sofia 62, Catania, 95123 Italy.

${ }^{21}$ North-West University, Centre for Space Research, Private Bag X6001, Potchefstroom, 2520 South Africa.

${ }^{22}$ Instituto Español de Oceanografía, Unidad Mixta IEO-UPV, C/ Paranimf, 1, Gandia, 46730 Spain.

${ }^{23}$ University Mohammed I, Faculty of Sciences, BV Mohammed VI, B.P. 717, R.P. 60000 Oujda, Morocco.

${ }^{24}$ Subatech, IMT Atlantique, IN2P3-CNRS, Université de Nantes, 4 rue Alfred Kastler - La Chantrerie, Nantes, BP 2072244307 France.

${ }^{25}$ Università di Salerno e INFN Gruppo Collegato di Salerno, Dipartimento di Matematica, Via Giovanni Paolo II 132, Fisciano, 84084 Italy.

${ }^{26}$ ISS, Atomistilor 409, Măgurele, RO-077125 Romania.

${ }^{27}$ University of Amsterdam, Institute of Physics/IHEF, PO Box 94216, Amsterdam, 1090 GE Netherlands.

${ }^{28}$ TNO, Technical Sciences, PO Box 155, Delft, 2600 AD Netherlands.

${ }^{29}$ Università degli Studi della Campania "Luigi Vanvitelli", Dipartimento di Matematica e Fisica, viale Lincoln 5, Caserta, 81100 Italy.

${ }^{30}$ Università La Sapienza, Dipartimento di Fisica, Piazzale Aldo Moro 2, Roma, 00185 Italy.

${ }^{31}$ Università di Bologna, Dipartimento di Ingegneria dell'Energia Elettrica e dell'Informazione "Guglielmo Marconi", Via dell’Università 50, Cesena, 47521 Italia.

${ }^{32}$ Cadi Ayyad University, Physics Department, Faculty of Science Semlalia, Av. My Abdellah, P.O.B. 2390, Marrakech, 40000 Morocco.

${ }^{33}$ University of the Witwatersrand, School of Physics, Private Bag 3, Johannesburg, Wits 2050 South Africa.

${ }^{34}$ Università di Catania, Dipartimento di Fisica e Astronomia "Ettore Majorana", Via Santa Sofia 64, Catania, 95123 Italy.

${ }^{35}$ INFN, LNF, Via Enrico Fermi, 40, Frascati, 00044 Italy.

${ }^{36}$ INFN, Sezione di Bari, via Orabona, 4, Bari, 70125 Italy.

${ }^{37}$ International Centre for Radio Astronomy Research, Curtin University, Bentley, WA 6102, Australia.

${ }^{38}$ University of Bari, Via Amendola 173, Bari, 70126 Italy.

${ }^{39}$ University Würzburg, Emil-Fischer-Straße 31, Würzburg, 97074 Germany.

${ }^{40}$ Western Sydney University, School of Computing, Engineering and Mathematics, Locked Bag 1797, Penrith, NSW 2751 Australia.

${ }^{41}$ Università di Salerno e INFN Gruppo Collegato di Salerno, Dipartimento di Fisica, Via Giovanni Paolo II 132, Fisciano, 84084 Italy.

${ }^{42}$ Università di Genova, Via Dodecaneso 33, Genova, 16146 Italy.

${ }^{43}$ Normandie Univ, ENSICAEN, UNICAEN, CNRS/IN2P3, LPC Caen, LPCCAEN, 6 boulevard Maréchal Juin, Caen, 14050 France.

${ }^{44}$ University of Granada, Dpto. de Física Teórica y del Cosmos \& C.A.F.P.E., 18071 Granada, Spain.

${ }^{45}$ NIOZ (Royal Netherlands Institute for Sea Research), PO Box 59, Den Burg, Texel, 1790 AB, the Netherlands.

${ }^{46}$ Tbilisi State University, Department of Physics, 3, Chavchavadze Ave., Tbilisi, 0179 Georgia.

${ }^{47}$ Leiden University, Leiden Institute of Physics, PO Box 9504, Leiden, 2300 RA Netherlands.

${ }^{48}$ National Centre for Nuclear Research, 02-093 Warsaw, Poland.

${ }^{49}$ Institut Universitaire de France, 1 rue Descartes, Paris, 75005 France.

${ }^{50}$ University of Johannesburg, Department Physics, PO Box 524, Auckland Park, 2006 South Africa.

${ }^{51}$ Eberhard Karls Universität Tübingen, Institut für Astronomie und Astrophysik, Sand 1, Tübingen, 72076 Germany.

${ }^{52}$ Università di Pisa, Dipartimento di Fisica, Largo Bruno Pontecorvo 3, Pisa, 56127 Italy.

${ }^{53}$ Laboratoire Univers et Particules de Montpellier, Place Eugène Bataillon - CC 72, Montpellier Cédex 05, 34095 France.

${ }^{54}$ Friedrich-Alexander-Universität Erlangen-Nürnberg, Remeis Sternwarte, Sternwartstraße 7, 96049 Bamberg, Germany.

${ }^{55}$ Université de Haute Alsace, 68100 Mulhouse Cedex, France.

${ }^{56}$ University of Münster, Institut für Kernphysik, Wilhelm-Klemm-Str. 9, Münster, 48149 Germany.

${ }^{57}$ Utrecht University, Department of Physics and Astronomy, PO Box 80000, Utrecht, 3508 TA Netherlands.

${ }^{58}$ INFN, CNAF, v.le C. Berti-Pichat, 6/2, Bologna, 40127 Italy. 